\title{
THE EFFECTS OF 1-HYDRAZINOPHTHALAZINE UPON CORO- NARY HEMODYNAMICS AND MYOCARDIAL OXYGEN METABOLISM IN ESSENTIAL HYPERTENSION ${ }^{2}$
}

\author{
By GEORGE G. ROWE, 2 JOHN H. HUSTON, 3 GEORGE M. MAXWELL, ARVIN B. \\ WEINSTEIN, HERMAN TUCHMAN, AND CHARLES W. CRUMPTON \\ WITH THE TECHNICAL ASSISTANCE OF BERYL E. WELCH AND \\ AUDREY L. PETERSON
}

(From the Cardiovascular Research Laboratory and the Department of Medicine, University of Wisconsin Medical School and the University Hospitals, Madison, Wis.)

(Submitted for publication December 6, 1954; accepted December 29, 1954)

Since it has been shown that the most deleterious effects of hypertension occur in cerebral, renal and coronary vascular beds, it seems wise to evaluate these circulatory regions in relation to the effect of anti-hypertensive drugs. Reports of the hemodynamic effects of Hydralazine ${ }^{4}$ on the renal $(1-3)$ and cerebral $(4,5)$ circulations are already available, but comparable information is lacking in relation to the coronary circulation. Observations concerning coronary flow after the administration of Hydralazine are particularly desirable since it is known to precipitate angina pectoris in some patients (6). The current report concerns an investigation of the cardiovascular hemodynamic effects of Hydralazine in subjects with arterial hypertension.

\section{MATERIAL AND METHOD}

Eleven hypertensive subjects from general medical wards were studied. Five of these subjects served as controls with determination of the various cardiac functions before and after administration of $1 \mathrm{cc}$. of saline solution.

Six served as experimental subjects with the same determinations made before and after Hydralazine. The determination of hemodynamic functions in this study was by the usual methods and has been reported previously from this laboratory (7). The determination of coronary blood flow was by the nitrous oxide saturation method (8). In each patient, cardiac output was deter-

1 This work was supported in part by grants from the National Heart Institute, United States Public Health Service, the Wisconsin Alumni Research Foundation, and the Wisconsin Heart Association.

2 This work was done during the tenure of a Research Fellowship of the American Heart Association.

8 This work was done during the tenure of a Research Fellowship of the Wisconsin Heart Association.

- Hydralazine was supplied by Ciba Pharmaceutical Products, Inc., Summit, N. J. mined by the Fick principle. The catheter was then withdrawn from the pulmonary artery and placed in the coronary sinus for coronary blood flow determination. Approximately one-half hour after administration of either Hydralazine or saline through the cardiac catheter coronary blood flow determination was repeated. The catheter was then placed in the pulmonary artery and cardiac output was again determined. Thus each patient served as his own control, and the five patients who received the saline placebo served as controls for the method and procedure.

\section{RESULTS}

The results are seen in Table I. Significant changes occurred in the placebo (saline) group in only two of the determinations made. One was a slight fall in the body respiratory quotient and the other a slight fall in the mixed venous oxygen content. The reason for these two changes is not apparent, but it is of interest that their significance depends upon the consistency rather than the magnitude of change.

After administration of Hydralazine, hemodynamic changes occurred similar to those already reported by others $(1,3)$ and by this laboratory (7). These changes included an increase in heart rate, a fall in systemic blood pressure and peripheral arterial resistance, a decreased arterial and mixed venous carbon dioxide content, and a smaller arteriovenous carbon dioxide difference. The change in cardiac index in this series was variable, as has already been reported (7), but the mean average tended to increase following Hydralazine administration. Total body carbon dioxide production and oxygen consumption were unchanged and the respiratory quotient was constant. The hemodynamic data concerning these five patients were reported previously exclusive of the coronary metabolic data (7). Hydralazine 
produced an increase in coronary blood flow in five out of six subjects, the over-all increase being 34 per cent. Simultaneously, with the decrease in perfusion pressure, there was a fall in coronary vascular resistance ( -35 per cent). The increased coronary blood flow was accompanied by a decrease in the arterial-coronary sinus oxygen difference, such that left ventricular oxygen utilization was unchanged.

\section{DISCUSSION}

This study reveals that Hydralazine decreases coronary vascular resistance to such a degree that an increase in flow occurs in spite of the reduced perfusion pressure. Simultaneously, the arteriovenous oxygen difference across the myocardium narrows so that the left ventricular oxygen consumption per $100 \mathrm{~g}$. remains unchanged. It was noted previously that the blood carbon dioxide levels were altered by the administration of $\mathrm{Hy}$ dralazine (7), and these observations show that the changes are extended to the coronary circulation where a significant fall in coronary sinus carbon dioxide level occurs. These were correlated previously with an increase in minute volume of respiration and a decrease in oxygen consumption per liter of ventilation. Similar changes occurred in this series but were not significant, probably because this group is smaller than the previous series.

The reason for the precipitation of angina by Hydralazine is not apparent from this study. In four out of five subjects the coronary blood flow per kg. meter of left ventricular work rose. Although no clear relationship is noted here between left ventricular work and oxygen consumption it is interesting that in all but one subject the number of cc. of available oxygen per $\mathrm{kg}$. meter of left ventricular work [ (Art. $\left.\mathrm{O}_{2} \times \mathrm{CBF}\right) \div \mathrm{L}$.V. Work] increased after Hydralazine. In confirmation of these ratios the coronary sinus blood oxygen content rose in each instance and therefore the myocardial oxygen tension must have been higher after Hydralazine than it was before. Four out of five patients eliminated more $\mathrm{CO}_{2}$ from the heart per $\mathrm{kg}$. meter of left ventricular work after Hydralazine but it cannot be stated definitely whether this was due to the falling level of $\mathrm{CO}_{2}$ in the blood and heart. In view of these findings it may be speculated that angina is precipitated only in those patients who have a fixed coronary vascular resistance, which cannot compensate for the decrease in perfusion pressure and therefore have a reduction in coronary flow. We did not observe such a response in this series. The possibility also exists that Hydralazine specifically alters myocardial oxidative metabolism so that available oxygen is not utilized and angina is precipitated by a metabolic block.

\section{SUMMARY AND CONCLUSIONS}

1. Coronary blood flow and myocardial oxygen metabolism have been studied in eleven hypertensive subjects.

2. Compared with the five placebo studies, there were in the six Hydralazine studies statistically significant changes in that coronary blood flow increased by 34 per cent, coronary vascular resistance decreased by 35 per cent, and the arterialcoronary sinus oxygen difference decreased by 27 per cent due to an 81 per cent increase in the coronary sinus oxygen content.

3. The cardiac metabolic rate for oxygen remained unchanged.

\section{REFERENCES}

1. Moyer, J. H., Handley, C. A., and Huggins, R. A., Some pharmacodynamic effects of 1-hydrazinophthalazine (C-5968) with particular reference to renal function and cardiovascular response. $\mathrm{J}$. Pharmacol. \& Exper. Therap., 1951, 103, 368.

2. Crosley, A. P., Jr., Rowe, G. G., and Crumpton, C. W., The hemodynamic and metabolic response of the human hypertensive kidney to a standard dose of 1-hydrazinophthalazine (Hydralazine). J. Lab. \& Clin. Med., 1954, 44, 104.

3. Wilkinson, E. L., Backman, H., and Hecht, H. H., Cardiovascular and renal adjustments to a hypotensive agent (1-hydrazinophthalazine: Ciba BA-5968: Apresoline). J. Clin. Invest., 1952, 31, 872.

4. Hafkenschiel, J. H., and Friedland, C. K., The effect of 1-hydrazinophthalazine on cerebral blood flow, vascular resistance, oxygen uptake and jugular oxygen tension in hypertensive subjects. J. Clin. Invest., 1953, 32, 655.

5. Crumpton, C. W., Rowe, G. G., Crosley, A. P., Jr., Maxwell, G. M., and Huston, J. H., Cardiovascular, cerebral, and renal hemodynamic and metabolic adjustments to 1-hydrazinophthalazine in essential hypertension. Proc. Cent. Soc. for Clinical Re- 
TABLE I

\begin{tabular}{|c|c|c|c|c|c|c|c|c|c|c|c|c|c|c|c|c|c|}
\hline Patient & Age & Sex & $\begin{array}{c}\text { Ht. } \\
\text { in } \\
c m .\end{array}$ & 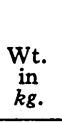 & $\begin{array}{c}\mathrm{SA}^{1} \\
\text { in } \\
\boldsymbol{M}^{2}\end{array}$ & $\begin{array}{l}\text { Card. } \\
\text { rate }\end{array}$ & $\begin{array}{c}\text { Syst.2 } \\
\text { BP } \\
\text { mean } \\
m_{m .} . \\
H g\end{array}$ & $\underset{\substack{\mathrm{BP} \\
\text { mean } \\
\mathrm{Hg}}}{\mathrm{PA}^{\mathrm{s}}}$ & $\begin{array}{l}\text { Min.4 } \\
\text { vol. } \\
\text { resp. } \\
L .\end{array}$ & $\begin{array}{c}\text { Os } \\
\text { cons. } \\
c c . / \\
\text { min. }\end{array}$ & $\begin{array}{c}\mathrm{O}_{2}^{6} \\
\text { cons./ } \\
\text { L. } \\
\text { vent. }\end{array}$ & $\begin{array}{c}\text { Art.7 } \\
\mathrm{O}_{2} \\
\text { Vol. } \\
\%\end{array}$ & $\begin{array}{c}\text { Mixed } \\
\text { venous } \\
\mathrm{O}_{2} \\
\mathrm{Vol}^{3} . \\
\% \\
\%\end{array}$ & $\begin{array}{c}\Delta \text { Art. } \\
\text { m. ven. } \\
\mathrm{O}_{2} \\
V_{2} \text {. } \\
\% \\
\%\end{array}$ & $\begin{array}{c}\mathrm{CS}^{10} \\
\mathrm{O}_{2} \\
\text { Vol. } \\
\%\end{array}$ & $\begin{array}{c}\Delta \text { Art. }^{11} \\
\text { CSOO }_{2} \\
\text { Vol. } \\
\%\end{array}$ & $\begin{array}{l}\text { Art.12 } \\
\mathrm{CO}_{2} \\
\text { Vol. } \\
\% \\
\end{array}$ \\
\hline \multicolumn{18}{|c|}{ Part I-Control series } \\
\hline T. 0. & 35 & $\mathbf{M}$ & 168 & 87 & 2.0 & $\begin{array}{l}83 \\
82\end{array}$ & $\begin{array}{l}125 \\
142\end{array}$ & $\begin{array}{l}15 \\
17\end{array}$ & $\begin{array}{l}7.2 \\
6.7\end{array}$ & $\begin{array}{l}314 \\
284\end{array}$ & $\begin{array}{l}44 \\
42\end{array}$ & $\begin{array}{l}21.2 \\
21.2\end{array}$ & $\begin{array}{l}16.8 \\
16.9\end{array}$ & $\begin{array}{l}4.4 \\
4.3\end{array}$ & $\begin{array}{l}7.4 \\
7.6\end{array}$ & $\begin{array}{l}13.1 \\
13.6\end{array}$ & $\begin{array}{l}41.4 \\
41.5\end{array}$ \\
\hline C. S. & 31 & $\mathbf{M}$ & 168 & 70 & 1.8 & $\begin{array}{l}81 \\
81\end{array}$ & $\begin{array}{l}129 \\
132\end{array}$ & $\begin{array}{l}7 \\
6\end{array}$ & $\begin{array}{l}5.0 \\
4.7\end{array}$ & $\begin{array}{l}228 \\
218\end{array}$ & $\begin{array}{l}46 \\
46\end{array}$ & $\begin{array}{l}18.7 \\
18.3\end{array}$ & $\begin{array}{l}14.3 \\
13.4\end{array}$ & $\begin{array}{l}4.4 \\
4.9\end{array}$ & $\begin{array}{l}7.3 \\
7.2\end{array}$ & $\begin{array}{l}11.4 \\
11.1\end{array}$ & $\begin{array}{l}42.9 \\
42.3\end{array}$ \\
\hline H. J. & 45 & $\mathbf{M}$ & 170 & 59 & 1.7 & $\begin{array}{l}89 \\
84\end{array}$ & $\begin{array}{l}162 \\
166\end{array}$ & $\begin{array}{l}17 \\
19\end{array}$ & $\begin{array}{l}6.2 \\
6.0\end{array}$ & $\begin{array}{l}236 \\
247\end{array}$ & $\begin{array}{l}38 \\
41\end{array}$ & $\begin{array}{l}18.4 \\
18.6\end{array}$ & $\begin{array}{l}13.2 \\
12.3\end{array}$ & $\begin{array}{l}5.2 \\
6.3\end{array}$ & $\begin{array}{l}6.9 \\
6.3\end{array}$ & $\begin{array}{l}11.5 \\
12.3\end{array}$ & $\begin{array}{l}48.5 \\
46.4\end{array}$ \\
\hline H. P. & 46 & $\mathbf{M}$ & 171 & 75 & 1.9 & $\begin{array}{l}65 \\
69\end{array}$ & $\begin{array}{l}154 \\
158\end{array}$ & $\begin{array}{l}16 \\
13\end{array}$ & $\begin{array}{l}5.9 \\
6.0\end{array}$ & $\begin{array}{l}290 \\
293\end{array}$ & $\begin{array}{l}49 \\
49\end{array}$ & $\begin{array}{l}17.0 \\
16.4\end{array}$ & $\begin{array}{l}12.1 \\
11.3\end{array}$ & $\begin{array}{l}4.9 \\
5.1\end{array}$ & $\begin{array}{l}6.7 \\
6.9\end{array}$ & $\begin{array}{r}10.3 \\
9.4\end{array}$ & $\begin{array}{l}50.0 \\
49.5\end{array}$ \\
\hline G. D. & 57 & $\mathbf{M}$ & 165 & 80 & 1.9 & $\begin{array}{l}83 \\
79\end{array}$ & $\begin{array}{l}128 \\
139\end{array}$ & $\begin{array}{l}14 \\
14\end{array}$ & $\begin{array}{l}7.7 \\
9.8\end{array}$ & $\begin{array}{l}253 \\
277\end{array}$ & $\begin{array}{l}33 \\
28\end{array}$ & $\begin{array}{l}20.1 \\
19.4\end{array}$ & $\begin{array}{l}15.0 \\
14.8\end{array}$ & $\begin{array}{l}5.1 \\
4.6\end{array}$ & $\begin{array}{l}5.8 \\
5.8\end{array}$ & $\begin{array}{l}14.3 \\
13.6\end{array}$ & $\begin{array}{l}47.6 \\
42.8\end{array}$ \\
\hline $\begin{array}{l}\text { 1st ave. } \\
\text { SD† }\end{array}$ & 43 & & & & & $\begin{array}{l}80 \\
\pm 9\end{array}$ & $\begin{array}{r}140 \\
\pm 19\end{array}$ & $\begin{array}{l}14 \\
\pm 4\end{array}$ & $\begin{array}{r}6.4 \\
\pm 1.1\end{array}$ & $\begin{array}{l}264 \\
\pm 37\end{array}$ & $\begin{array}{l}42 \\
\pm 6\end{array}$ & $\begin{array}{r}18.9 \\
\pm 1.4\end{array}$ & $\begin{array}{l}14.3 \\
\pm 1.8\end{array}$ & $\begin{array}{r}4.8 \\
\pm 0.4\end{array}$ & $\begin{array}{r}6.8 \\
\pm 0.6\end{array}$ & $\begin{array}{r}12.1 \\
\pm 1.6\end{array}$ & $\begin{array}{r}46.1 \\
\pm 3.7\end{array}$ \\
\hline$\underset{\text { SDt }}{\text { 2nd ave. }}$ & & & & & & $\begin{array}{r}79 \\
\pm 6\end{array}$ & $\begin{array}{r}147 \\
\pm 14\end{array}$ & $\begin{array}{r}14 \\
\pm 5\end{array}$ & $\begin{array}{r}6.6 \\
\pm 1.9\end{array}$ & $\begin{array}{r}264 \\
\pm 31\end{array}$ & $\begin{array}{r}41 \\
\pm 8\end{array}$ & $\begin{array}{r}18.8 \\
\pm 1.7\end{array}$ & $\begin{array}{r}13.7 \\
\pm 2.2\end{array}$ & $\begin{array}{r}5.1 \\
\pm 0.8\end{array}$ & $\begin{array}{r}6.8 \\
\pm 0.7\end{array}$ & $\begin{array}{r}12.0 \\
\pm 1.8\end{array}$ & $\begin{array}{r}44.5 \\
\pm 3.4\end{array}$ \\
\hline $\begin{array}{l}\text { Change } \\
\text { \% Change } \\
\text { P value }\end{array}$ & & & & & & $\begin{array}{l}-1 \\
-1 \%\end{array}$ & $\begin{array}{l}+7 \\
+5 \%\end{array}$ & $\begin{array}{l}\mathbf{0} \\
\mathbf{0}\end{array}$ & $\begin{array}{l}+0.2 \\
+3 \%\end{array}$ & $\begin{array}{l}\mathbf{0} \\
\mathbf{0} \%\end{array}$ & $\begin{array}{l}-1 \\
-2 \%\end{array}$ & $\begin{array}{l}-0.1 \\
-1 \%\end{array}$ & $\begin{array}{l}-0.6 \\
-5 \% \\
<0.05\end{array}$ & $\begin{array}{l}+0.3 \\
+7 \%\end{array}$ & $\begin{array}{l}\mathbf{0} \\
\mathbf{0}\end{array}$ & $\begin{array}{l}-0.1 \\
-1 \%\end{array}$ & $\begin{array}{l}-1.6 \\
-3 \%\end{array}$ \\
\hline \multicolumn{18}{|c|}{ Part II-Hydralazine series } \\
\hline H. J. & 31 & $\mathbf{M}$ & 176 & 76 & 1.9 & $\begin{array}{r}92 \\
128\end{array}$ & $\begin{array}{l}119 \\
117\end{array}$ & $\begin{array}{l}6 \\
9\end{array}$ & $\begin{array}{l}9.4 \\
7.3\end{array}$ & $\begin{array}{l}269 \\
351\end{array}$ & $\begin{array}{l}29 \\
48\end{array}$ & $\begin{array}{l}20.3 \\
21.3\end{array}$ & $\begin{array}{l}15.9 \\
16.5\end{array}$ & $\begin{array}{l}4.4 \\
4.8\end{array}$ & $\begin{array}{r}6.1 \\
11.2\end{array}$ & $\begin{array}{r}14.2 \\
8.9\end{array}$ & $\begin{array}{l}43.0 \\
39.1\end{array}$ \\
\hline R. J. & 47 & $\mathbf{M}$ & 173 & 79 & 1.9 & $\begin{array}{l}78 \\
98\end{array}$ & $\begin{array}{l}136 \\
128\end{array}$ & $\begin{array}{l}14 \\
11\end{array}$ & $\begin{array}{l}5.7 \\
7.6\end{array}$ & $\begin{array}{l}280 \\
303\end{array}$ & $\begin{array}{l}49 \\
40\end{array}$ & $\begin{array}{l}18.2 \\
18.7\end{array}$ & $\begin{array}{l}13.5 \\
15.3\end{array}$ & $\begin{array}{l}4.7 \\
3.4\end{array}$ & $\begin{array}{r}5.4 \\
11.9\end{array}$ & $\begin{array}{r}12.8 \\
6.8\end{array}$ & $\begin{array}{l}45.3 \\
39.7\end{array}$ \\
\hline A. $\mathrm{R}$. & 63 & $\mathbf{F}$ & 166 & 73 & 1.8 & $\begin{array}{l}85 \\
87\end{array}$ & $\begin{array}{r}128 \\
75\end{array}$ & $\begin{array}{l}12 \\
12\end{array}$ & $\begin{array}{r}6.6 \\
10.6\end{array}$ & $\begin{array}{l}228 \\
207\end{array}$ & $\begin{array}{l}35 \\
20\end{array}$ & $\begin{array}{l}15.5 \\
15.3\end{array}$ & $\begin{array}{l}11.0 \\
11.2\end{array}$ & $\begin{array}{l}4.5 \\
4.1\end{array}$ & $\begin{array}{l}4.7 \\
5.8\end{array}$ & $\begin{array}{r}10.8 \\
9.5\end{array}$ & $\begin{array}{l}36.8 \\
33.1\end{array}$ \\
\hline J.P. & 32 & $\mathbf{F}$ & 166 & 57 & 1.6 & $\begin{array}{l}100 \\
102\end{array}$ & $\begin{array}{l}168 \\
105\end{array}$ & $\begin{array}{l}4 \\
7\end{array}$ & $\begin{array}{l}5.9 \\
7.6\end{array}$ & $\begin{array}{l}226 \\
196\end{array}$ & $\begin{array}{l}38 \\
26\end{array}$ & $\begin{array}{l}19.3 \\
19.0\end{array}$ & $\begin{array}{l}14.1 \\
14.3\end{array}$ & $\begin{array}{l}5.2 \\
4.7\end{array}$ & $\begin{array}{l}3.6 \\
7.8\end{array}$ & $\begin{array}{l}15.7 \\
11.2\end{array}$ & $\begin{array}{l}44.7 \\
39.7\end{array}$ \\
\hline J. V. & 62 & $\mathbf{M}$ & 160 & 91 & 1.9 & $\begin{array}{l}63 \\
75\end{array}$ & $\begin{array}{l}120 \\
109\end{array}$ & 二 & 二 & 二 & $=$ & $\begin{array}{l}21.7 \\
22.0\end{array}$ & $=$ & $=$ & $\begin{array}{l}5.5 \\
9.5\end{array}$ & $\begin{array}{l}16.2 \\
12.5\end{array}$ & $\begin{array}{l}38.5 \\
36.2\end{array}$ \\
\hline Y. B. & 51 & F & 150 & 53 & 1.5 & $\begin{array}{r}78 \\
115\end{array}$ & $\begin{array}{l}157 \\
126\end{array}$ & $\begin{array}{r}10 \\
6\end{array}$ & $\begin{array}{l}3.9 \\
4.2\end{array}$ & $\begin{array}{l}201 \\
186\end{array}$ & $\begin{array}{l}51 \\
44\end{array}$ & $\begin{array}{l}17.9 \\
18.1\end{array}$ & $\begin{array}{l}13.6 \\
13.6\end{array}$ & $\begin{array}{l}4.3 \\
4.5\end{array}$ & $\begin{array}{l}3.6 \\
5.8\end{array}$ & $\begin{array}{l}14.3 \\
12.3\end{array}$ & $\begin{array}{l}50.0 \\
45.9\end{array}$ \\
\hline $\begin{array}{l}\text { Cont.ave. } \\
\text { S.D.t }\end{array}$ & 48 & & & & & $\begin{array}{r}83 \\
\pm 13\end{array}$ & $\begin{array}{r}138 \\
\pm 20\end{array}$ & $\begin{array}{r}9 \\
\pm 4\end{array}$ & $\begin{array}{r}6.3 \\
\pm 2.0\end{array}$ & $\begin{array}{r}241 \\
\pm 33\end{array}$ & $\begin{array}{r}40 \\
\pm 9\end{array}$ & $\begin{array}{r}18.8 \\
\pm 2.1\end{array}$ & $\begin{array}{r}13.6 \\
\pm 1.7\end{array}$ & $\begin{array}{r}4.6 \\
\pm 0.4\end{array}$ & $\begin{array}{r}4.8 \\
\pm 1.0\end{array}$ & $\begin{array}{r}14.0 \\
\pm 2.0\end{array}$ & $\begin{array}{r}43.1 \\
\pm 4.8\end{array}$ \\
\hline $\begin{array}{c}\text { Drug ave. } \\
\text { S.D. } t\end{array}$ & & & & & & $\begin{array}{l}101 \\
\pm 19\end{array}$ & $\begin{array}{r}110 \\
\pm 19\end{array}$ & $\begin{array}{r}9 \\
\pm 3\end{array}$ & $\begin{array}{r}7.5 \\
\pm 2.3\end{array}$ & $\begin{array}{r}249 \\
\pm 74\end{array}$ & $\begin{array}{r}36 \\
\pm 12\end{array}$ & $\begin{array}{r}19.1 \\
\pm 2.4\end{array}$ & $\begin{array}{r}14.2 \\
\pm 2.0\end{array}$ & $\begin{array}{r}4.3 \\
\pm 0.6\end{array}$ & $\begin{array}{r}8.7 \\
\pm 2.6\end{array}$ & $\begin{array}{r}10.2 \\
\pm 3.8\end{array}$ & $\begin{array}{r}39.0 \\
\pm 4.3\end{array}$ \\
\hline $\begin{array}{l}\text { Change } \\
\text { \% Change } \\
\text { P value }\end{array}$ & & & & & & $\begin{array}{l}+18 \\
+22 \% \\
<0.05\end{array}$ & $\begin{array}{l}-28 \\
-20 \% \\
<0.05\end{array}$ & $\begin{array}{l}\mathbf{0} \\
\mathbf{0} \\
-\end{array}$ & $\begin{array}{l}+1.2 \\
+19 \% \\
<0.3\end{array}$ & $\begin{array}{l}+8 \\
+3 \% \\
<0.8\end{array}$ & $\begin{array}{l}-4 \\
-10 \% \\
<0.5\end{array}$ & $\begin{array}{l}+0.3 \\
+2 \% \\
<0.3\end{array}$ & $\begin{array}{l}+0.6 \\
+1 \% \\
<.2\end{array}$ & $\begin{array}{l}-0.3 \\
<-7 \% \\
<0.4\end{array}$ & $\begin{array}{l}+3.9 \\
+81 \% \\
<0.01\end{array}$ & $\begin{array}{l}-3.8 \\
-27 \% \\
<0.01\end{array}$ & $\begin{array}{l}-4.1 \\
-10 \% \\
<0.01\end{array}$ \\
\hline
\end{tabular}

1 Body surface area in square meters.

2 Systemic arterial blood pressure mean in $\mathrm{mm}$. $\mathrm{Hg}$.

3 Pulmonary arterial blood pressure mean in $\mathrm{mm}$. Hg.

Minute volume of air exhaled in litres.

cc. of $\mathrm{O}_{2}$ consumed per minute.

cc. of $\mathrm{O}_{2}$ consumed per litre of exhaled air.

7 Arterial $\mathrm{O}_{2}$ content in vol. per $100 \mathrm{cc}$.

- Mixed venous (pulmonary arterial) $\mathrm{O}_{2}$ content in vol. per $100 \mathrm{cc}$.
Arterial-mixed venous $\mathrm{O}_{2}$ difference in cc. per $100 \mathrm{cc}$.

${ }_{10}$ Coronary sinus $\mathrm{O}_{2}$ content in vol. per $100 \mathrm{cc}$.

11 Arterial-coronary sinus $\mathrm{O}_{2}$ difference in vol. per $100 \mathrm{cc}$.

12 Arterial $\mathrm{CO}_{2}$ content in vol. per $100 \mathrm{cc}$.

13 Mixed venous (pulm. arterial) $\mathrm{CO}_{2}$ in vol. per $100 \mathrm{cc}$.

14 Arterial-mixed venous $\mathrm{CO}_{2}$ difference in vol. per $100 \mathrm{cc}$.

is Coronary sinus $\mathrm{CO}_{2}$ content in vol. per $100 \mathrm{cc}$. 
TABLE I

\begin{tabular}{|c|c|c|c|c|c|c|c|c|c|c|c|c|c|c|c|c|}
\hline $\begin{array}{c}\text { Mixed }^{18} \\
\text { venous } \\
\mathrm{CO}_{2} \\
\text { Vol. } \\
\%\end{array}$ & $\begin{array}{c}\Delta \text { Art.14 } \\
\text { venous } \\
\mathrm{CO}_{2} \\
\text { Vol. } \\
\%\end{array}$ & $\begin{array}{l}\mathrm{CS} 15 \\
\mathrm{CO}_{2} \\
\text { Vol. } \\
\% 1\end{array}$ & $\begin{array}{l}\text { AArt.16 } \\
\text { C.S.S. } \\
\text { ÇO. } \\
\text { Vol. } \\
\%\end{array}$ & $\begin{array}{c}\mathrm{CO}_{2}^{17} \\
\text { prod./ } \\
\text { min. }\end{array}$ & $\begin{array}{c}\text { Cardiac } 18 \\
\text { index }\end{array}$ & $\begin{array}{l}\text { Totals } \\
\text { periph. } \\
\text { resist. }\end{array}$ & $\begin{array}{l}\text { Total } 20 \\
\text { pulm. } \\
\text { resist. }\end{array}$ & $\underset{\text { work }}{\text { L.V.21 }}$ & $\underset{\text { work }}{\text { R.V.22 }}$ & $\begin{array}{l}\text { Cor. } \\
\text { blood } \\
\text { flow }\end{array}$ & $\underset{\mathrm{O}_{2}}{\mathrm{CMR}}$ & C.V.R.25 & $\begin{array}{c}\text { Druges }_{\text {dose }} \\
\text { in } \\
m g .\end{array}$ & $\underset{\substack{\text { Drug } \\
\text { in } \\
\text { mg./ } \\
\text { kg. }}}{ }$ & $\begin{array}{c}\text { Timen } \\
\text { from } \\
\text { drug } \\
\text { to } \\
\text { study }\end{array}$ & $\begin{array}{l}\text { Body" } \\
\text { R.Q. }\end{array}$ \\
\hline \multicolumn{17}{|c|}{ Part I-Control series } \\
\hline $\begin{array}{l}45.5 \\
45.8\end{array}$ & $\begin{array}{l}4.1 \\
4.3\end{array}$ & $\begin{array}{l}52.3 \\
52.2\end{array}$ & $\begin{array}{l}10.9 \\
10.7\end{array}$ & $\begin{array}{l}245 \\
217\end{array}$ & $\begin{array}{l}3.8 \\
2.6\end{array}$ & $\begin{array}{l}1330 \\
2187\end{array}$ & $\begin{array}{l}155 \\
262\end{array}$ & $\begin{array}{l}12.8 \\
10.0\end{array}$ & $\begin{array}{l}1.5 \\
1.2\end{array}$ & $\begin{array}{l}67 \\
62\end{array}$ & $\begin{array}{l}8.8 \\
8.4\end{array}$ & $\begin{array}{l}1.9 \\
2.1\end{array}$ & Saline & 二 & $\overline{32}$ & $\begin{array}{l}0.78 \\
0.76\end{array}$ \\
\hline $\begin{array}{l}46.1 \\
44.9\end{array}$ & $\begin{array}{l}3.2 \\
2.6\end{array}$ & $\begin{array}{l}50.6 \\
50.0\end{array}$ & $\begin{array}{l}7.7 \\
7.7\end{array}$ & $\begin{array}{l}179 \\
163\end{array}$ & $\begin{array}{l}2.9 \\
2.5\end{array}$ & $\begin{array}{l}1989 \\
2370\end{array}$ & $\begin{array}{r}114 \\
99\end{array}$ & $\begin{array}{l}9.1 \\
8.0\end{array}$ & $\begin{array}{l}0.5 \\
0.3\end{array}$ & $\begin{array}{l}72 \\
63\end{array}$ & $\begin{array}{l}8.1 \\
9.4\end{array}$ & $\begin{array}{l}1.8 \\
2.1\end{array}$ & Saline & - & $\overline{24}$ & $\begin{array}{l}0.79 \\
0.78\end{array}$ \\
\hline $\begin{array}{l}52.6 \\
51.5\end{array}$ & $\begin{array}{l}4.1 \\
5.1\end{array}$ & $\begin{array}{l}57.3 \\
56.6\end{array}$ & $\begin{array}{r}8.8 \\
10.2\end{array}$ & $\begin{array}{l}197 \\
195\end{array}$ & $\begin{array}{l}2.7 \\
2.3\end{array}$ & $\begin{array}{l}2853 \\
3384\end{array}$ & $\begin{array}{l}293 \\
386\end{array}$ & $\begin{array}{r}10.0 \\
8.9\end{array}$ & $\begin{array}{l}1.0 \\
1.0\end{array}$ & $\begin{array}{l}81 \\
90\end{array}$ & $\begin{array}{r}9.3 \\
11.1\end{array}$ & $\begin{array}{l}2.1 \\
1.9\end{array}$ & Saline & 二 & $\overline{23}$ & $\begin{array}{l}0.84 \\
0.79\end{array}$ \\
\hline $\begin{array}{l}53.0 \\
53.1\end{array}$ & $\begin{array}{l}3.0 \\
3.6\end{array}$ & $\begin{array}{l}58.3 \\
56.9\end{array}$ & $\begin{array}{l}8.3 \\
7.4\end{array}$ & $\begin{array}{l}220 \\
216\end{array}$ & $\begin{array}{l}3.2 \\
3.1\end{array}$ & $\begin{array}{l}1990 \\
2161\end{array}$ & $\begin{array}{l}219 \\
174\end{array}$ & $\begin{array}{l}12.0 \\
12.5\end{array}$ & $\begin{array}{l}1.3 \\
1.0\end{array}$ & $\begin{array}{l}65 \\
69\end{array}$ & $\begin{array}{l}6.7 \\
6.5\end{array}$ & $\begin{array}{l}2.2 \\
2.1\end{array}$ & Saline & 二 & $\overline{23}$ & $\begin{array}{l}0.76 \\
0.74\end{array}$ \\
\hline $\begin{array}{l}51.5 \\
47.8\end{array}$ & $\begin{array}{l}3.9 \\
5.0\end{array}$ & $\begin{array}{l}\mathbf{5 8 . 5} \\
\mathbf{5 7 . 9}\end{array}$ & $\begin{array}{l}10.9 \\
15.1\end{array}$ & $\begin{array}{l}280 \\
274\end{array}$ & $\begin{array}{l}2.6 \\
3.1\end{array}$ & $\begin{array}{l}2042 \\
1862\end{array}$ & $\begin{array}{l}222 \\
194\end{array}$ & $\begin{array}{r}8.7 \\
11.2\end{array}$ & $\begin{array}{l}1.0 \\
1.2\end{array}$ & $\begin{array}{l}49 \\
50\end{array}$ & $\begin{array}{l}6.9 \\
6.7\end{array}$ & $\begin{array}{l}2.6 \\
2.7\end{array}$ & Saline & $\overline{\text { Ave. }}$ & $\begin{array}{r}\overline{34} \\
=27\end{array}$ & $\begin{array}{l}1.11 \\
0.99\end{array}$ \\
\hline $\begin{array}{r}49.7 \\
\pm 3.6\end{array}$ & $\begin{array}{r}3.7 \\
\pm 0.5\end{array}$ & $\begin{array}{r}55.4 \\
\pm 2.9\end{array}$ & $\begin{array}{r}3.7 \\
\pm 0.4\end{array}$ & $\begin{array}{r}224 \\
\pm 40\end{array}$ & $\begin{array}{r}3.0 \\
\pm 0.5\end{array}$ & $\begin{array}{r}2041 \\
\pm 541\end{array}$ & $\begin{array}{r}201 \\
\pm 60\end{array}$ & $\begin{array}{r}10.5 \\
\pm 1.8\end{array}$ & $\begin{array}{r}1.1 \\
\pm 0.4\end{array}$ & $\begin{array}{r}67 \\
\pm 14\end{array}$ & $\begin{array}{r}8.0 \\
\pm 1.1\end{array}$ & $\begin{array}{r}2.1 \\
\pm 0.3\end{array}$ & 二 & 二 & 二 & $\begin{array}{r}0.86 \\
\pm 0.14\end{array}$ \\
\hline $\begin{array}{r}48.6 \\
\pm 3.6\end{array}$ & $\begin{array}{r}4.1 \\
\pm 0.8\end{array}$ & $\begin{array}{r}54.7 \\
\pm 3.4\end{array}$ & $\begin{array}{r}4.1 \\
\pm 1.0\end{array}$ & $\begin{array}{r}213 \\
\pm 41\end{array}$ & $\begin{array}{r}2.7 \\
\pm 0.4\end{array}$ & $\begin{array}{r}2393 \\
\pm 583\end{array}$ & $\begin{array}{r}223 \\
\pm 108\end{array}$ & $\begin{array}{r}10.1 \\
\pm 1.8\end{array}$ & $\begin{array}{r}0.9 \\
\pm 0.4\end{array}$ & $\begin{array}{r}67 \\
\pm 15\end{array}$ & $\begin{array}{r}8.4 \\
\pm 1.9\end{array}$ & $\begin{array}{r}2.2 \\
\pm 0.3\end{array}$ & 二 & 二 & 二 & $\begin{array}{r}0.81 \\
\pm 0.10\end{array}$ \\
\hline $\begin{array}{l}-1.1 \\
-2 \%\end{array}$ & $\begin{array}{r}+0.4 \\
+11 \%\end{array}$ & $\begin{array}{l}-0.7 \\
-1 \%\end{array}$ & $\begin{array}{r}+0.4 \\
+11 \%\end{array}$ & $\frac{-11}{-5 \%}$ & $\begin{array}{r}-0.3 \\
-10 \%\end{array}$ & $\begin{array}{l}+352 \\
+17 \%\end{array}$ & $\begin{array}{l}+22 \\
+11 \%\end{array}$ & $\begin{array}{l}-0.4 \\
-4 \%\end{array}$ & $\begin{array}{c}-0.2 \\
-18 \%\end{array}$ & $\stackrel{\mathbf{0}}{\mathbf{0} \%}$ & $\begin{array}{l}+0.4 \\
+5 \%\end{array}$ & $\begin{array}{l}+0.1 \\
+5 \%\end{array}$ & - & 二 & 二 & $\begin{array}{l}-0.05 \\
-6 \% \\
<0.01\end{array}$ \\
\hline \multicolumn{17}{|c|}{ Part II-Hydralasine series } \\
\hline $\begin{array}{l}45.7 \\
40.7\end{array}$ & $\begin{array}{l}2.7 \\
1.6\end{array}$ & $\begin{array}{l}45.2 \\
43.3\end{array}$ & $\begin{array}{l}2.2 \\
4.2\end{array}$ & $\begin{array}{l}313 \\
221\end{array}$ & $\begin{array}{l}3.3 \\
6.0\end{array}$ & $\begin{array}{r}1554 \\
823\end{array}$ & $\begin{array}{l}83 \\
60\end{array}$ & $\begin{array}{r}9.9 \\
17.9\end{array}$ & $\begin{array}{l}0.5 \\
1.3\end{array}$ & $\begin{array}{r}70 \\
110\end{array}$ & $\begin{array}{r}10.0 \\
9.8\end{array}$ & $\begin{array}{l}1.7 \\
1.0\end{array}$ & 19 & $\underline{0.25}$ & 30 & $\begin{array}{l}1.16 \\
0.63\end{array}$ \\
\hline $\begin{array}{l}48.1 \\
41.5\end{array}$ & $\begin{array}{l}2.8 \\
1.8\end{array}$ & $\begin{array}{l}52.6 \\
45.0\end{array}$ & $\begin{array}{l}7.3 \\
5.3\end{array}$ & $\begin{array}{l}200 \\
207\end{array}$ & $\begin{array}{l}3.1 \\
4.6\end{array}$ & $\begin{array}{l}1826 \\
1147\end{array}$ & $\begin{array}{r}189 \\
96\end{array}$ & $\begin{array}{l}11.0 \\
15.5\end{array}$ & $\begin{array}{l}1.1 \\
1.3\end{array}$ & $\begin{array}{r}76 \\
118\end{array}$ & $\begin{array}{l}9.7 \\
8.0\end{array}$ & $\begin{array}{l}1.8 \\
1.0\end{array}$ & 20 & $\underline{0.25}$ & 30 & $\begin{array}{l}0.71 \\
0.68\end{array}$ \\
\hline $\begin{array}{l}38.7 \\
35.8\end{array}$ & $\begin{array}{l}1.9 \\
2.7\end{array}$ & $\begin{array}{l}43.5 \\
43.8\end{array}$ & $\begin{array}{r}6.7 \\
10.7\end{array}$ & $\begin{array}{l}143 \\
159\end{array}$ & $\begin{array}{l}2.8 \\
2.8\end{array}$ & $\begin{array}{l}2015 \\
1189\end{array}$ & $\begin{array}{l}187 \\
184\end{array}$ & $\begin{array}{l}8.8 \\
5.1\end{array}$ & $\begin{array}{l}0.8 \\
0.8\end{array}$ & $\begin{array}{l}79 \\
71\end{array}$ & $\begin{array}{l}8.6 \\
6.8\end{array}$ & $\begin{array}{l}1.6 \\
1.2\end{array}$ & 18 & $\underline{0.25}$ & 30 & $\begin{array}{l}0.62 \\
0.77\end{array}$ \\
\hline $\begin{array}{l}48.4 \\
42.8\end{array}$ & $\begin{array}{l}3.7 \\
3.1\end{array}$ & $\begin{array}{l}55.8 \\
48.3\end{array}$ & $\begin{array}{r}11.1 \\
8.6\end{array}$ & $\begin{array}{l}166 \\
179\end{array}$ & $\begin{array}{l}2.7 \\
2.6\end{array}$ & $\begin{array}{l}3077 \\
2021\end{array}$ & $\begin{array}{r}81 \\
131\end{array}$ & $\begin{array}{l}9.9 \\
6.0\end{array}$ & $\begin{array}{l}0.3 \\
0.4\end{array}$ & $\begin{array}{l}119 \\
167\end{array}$ & $\begin{array}{l}18.7 \\
18.7\end{array}$ & $\begin{array}{l}1.4 \\
0.6\end{array}$ & 14 & $\underline{0.25}$ & 32 & $\begin{array}{l}0.73 \\
0.92\end{array}$ \\
\hline 二 & $\overline{-}$ & $\begin{array}{l}50.2 \\
44.8\end{array}$ & $\begin{array}{r}11.7 \\
8.6\end{array}$ & 二 & 二 & 二 & 二 & 二 & 二 & $\begin{array}{l}56 \\
72\end{array}$ & $\begin{array}{l}9.1 \\
7.8\end{array}$ & $\begin{array}{l}2.1 \\
1.5\end{array}$ & 15 & $\underline{0.17}$ & 32 & 二 \\
\hline $\begin{array}{l}53.5 \\
49.1\end{array}$ & $\begin{array}{l}3.5 \\
3.2\end{array}$ & $\begin{array}{l}59.8 \\
54.7\end{array}$ & $\begin{array}{l}9.8 \\
8.8\end{array}$ & $\begin{array}{l}145 \\
129\end{array}$ & $\begin{array}{l}3.2 \\
2.9\end{array}$ & $\begin{array}{l}2685 \\
2427\end{array}$ & $\begin{array}{l}160 \\
123\end{array}$ & $\begin{array}{r}10.0 \\
7.1\end{array}$ & $\begin{array}{l}0.6 \\
0.4\end{array}$ & $\begin{array}{r}98 \\
132\end{array}$ & $\begin{array}{l}14.1 \\
16.2\end{array}$ & $\begin{array}{l}1.6 \\
1.1\end{array}$ & 11 & $\underline{0.20}$ & 30 & $\begin{array}{l}0.72 \\
0.70\end{array}$ \\
\hline $\begin{array}{r}46.9 \\
\pm 2.0\end{array}$ & $\begin{array}{r}2.9 \\
\pm 0.7\end{array}$ & $\begin{array}{r}51.2 \\
\pm 6.2\end{array}$ & $\begin{array}{r}9.3 \\
\pm 1.5\end{array}$ & $\begin{array}{r}193 \\
\pm 71\end{array}$ & $\begin{array}{r}\mathbf{3 . 0} \\
\pm \mathbf{0 . 3}\end{array}$ & $\begin{array}{r}2231 \\
\pm 631\end{array}$ & $\begin{array}{r}160 \\
\pm 46\end{array}$ & $\begin{array}{r}9.9 \\
\pm 0.8\end{array}$ & $\begin{array}{r}0.7 \\
\pm 0.3\end{array}$ & $\begin{array}{r}83 \\
\pm 22\end{array}$ & $\begin{array}{r}11.7 \\
\pm 4.0\end{array}$ & $\begin{array}{r}1.7 \\
\pm 0.2\end{array}$ & 二 & 二 & 二 & $\begin{array}{r}0.79 \\
\pm 0.21\end{array}$ \\
\hline $\begin{array}{r}42.0 \\
\pm 4.3\end{array}$ & $\begin{array}{r}2.5 \\
\pm 0.7\end{array}$ & $\begin{array}{r}46.6 \\
\pm 4.3\end{array}$ & $\begin{array}{r}10.2 \\
\pm 3.1\end{array}$ & $\begin{array}{r}179 \\
\pm 37\end{array}$ & $\begin{array}{r}3.8 \\
\pm 1.5\end{array}$ & $\begin{array}{r}1521 \\
\pm 672\end{array}$ & $\begin{array}{r}119 \\
\pm 46\end{array}$ & $\begin{array}{r}10.3 \\
\pm 5.9\end{array}$ & $\begin{array}{r}0.8 \\
\pm 0.5\end{array}$ & $\begin{array}{r}112 \\
\pm 37\end{array}$ & $\begin{array}{r}11.2 \\
\pm 5.0\end{array}$ & $\begin{array}{r}1.1 \\
\pm 0.3\end{array}$ & 16 & $\underline{0.23}$ & 31 & $\begin{array}{r}0.74 \\
\pm 0.11\end{array}$ \\
\hline $\begin{array}{r}-4.9 \\
-10 \% \\
<0.01\end{array}$ & $\begin{array}{r}-0.4 \\
-14 \% \\
<0.05\end{array}$ & $\begin{array}{l}-4.6 \\
-8 \% \\
<0.02\end{array}$ & $\begin{array}{l}+0.9 \\
+9 \% \\
<0.5\end{array}$ & $\begin{array}{l}-14 \\
-7 \% \\
<0.6\end{array}$ & $\begin{array}{r}+0.8 \\
+27 \% \\
<0.3\end{array}$ & $\begin{array}{r}-710 \\
-32 \% \\
<0.01\end{array}$ & $\begin{array}{c}-41 \\
-26 \% \\
<0.3\end{array}$ & $\begin{array}{r}+0.4 \\
+4 \%\end{array}$ & $\begin{array}{r}+0.1 \\
+14 \% \\
<0.4\end{array}$ & $\begin{array}{l}+28 \\
+34 \% \\
<0.05\end{array}$ & $\begin{array}{l}-0.5 \\
-5 \% \\
<0.5\end{array}$ & $\begin{array}{r}-0.6 \\
-35 \% \\
<0.01\end{array}$ & $=$ & 二 & $=$ & $\frac{-0.05}{<0.8}$ \\
\hline
\end{tabular}

16 Arterial-coronary sinus $\mathrm{CO}_{2}$ difference in vol. per $100 \mathrm{cc}$. $17 \mathrm{CO}_{2}$ produced in cc. per minute.

is Cardiac index in litres per sq. meter body surface area per min. 10 Total systemic arterial resistance in dynes per $\mathrm{cm} . \rightarrow / \mathrm{sec}$.

20 Total pulmonary arterial resistance in dynes per $\mathrm{cm} .-\mathrm{sec}$.

21 Left ventricular work in $\mathrm{kg}$. meters per min.

22 Right ventricular work in $\mathbf{k g}$. meters per min.

search, 26th annual meeting. J. Lab. \& Clin. Med., 1953, 42, 797.

6. Wilkins, R. W., Combination of drugs in the treatment of essential hypertension. Mississippi Doctor, 1953, 30, 359.

7. Rowe, G. G., Huston, J. H., Maxwell, G. M., Crosley, A. P., Jr., and Crumpton, C. W., Hemodynamic effects of 1-hydrazinophthalazine in patients with
2 Coronary blood flow in cc. per $100 \mathrm{gm}$. myocardium per min.

x Cardiac. $\mathrm{O}_{2}$ utilization per $100 \mathrm{gm}$. per min.

s Coronary vascular resistance (MABP $\div$ cor. blood flow).

* Hydralazine dose given through cardiac cath.

nI Hydralazine dose in $\mathrm{mgm}$. per $\mathrm{kg}$. body weight.

* Body respiratory quotient.

$\uparrow$ Standard deviation from the mean.

arterial hypertension. J. Clin. Invest., 1955, 34, 115.

8. Bing, R. J., Hammond, M. M., Handelsman, J. C., Powers, S. R., Spencer, F. C., Eckenhoff, J. E., Goodale, W. T., Hafkenschiel, J. H., and Kety, S. S., The measurement of coronary blood flow, oxygen consumption, and efficiency of the left ventricle in man. Am. Heart J., 1949, 38, 1. 